\title{
CHANGEIT: TOWARD AN APP TO HELP CHILDREN WITH AUTISM COPE WITH CHANGES
}

\author{
Vivian Varnava, Aurora Constantin and Cristina Adriana Alexandru \\ University of Edinburgh, School of Informatics \\ 10 Crichton Street, Edinburgh EH89AB, United Kingdom
}

\begin{abstract}
The use of technology-based interventions for ameliorating Autism Spectrum Disorder (ASD) core deficits has been growing in popularity. However, limited technologies are available that can help children with autism (aged 6 to 11) cope with changes, and these do not typically incorporate the methods used or recommended by practitioners. Our project addresses this gap through the design, development and evaluation of a prototype app to support children with ASD overcome their difficulties with changes. We report on preliminary work in developing this app, in which we decided not to involve children with ASD before getting some evidence that the app may be useful and suitable for them. Therefore, the design at this stage was informed by the research literature and design studies involving typically developing (TD) children, practitioners and researchers. The evaluation studies revealed that: 1) the app is easy to use; 2) the activities are perceived as fun and engaging; 3 ) the app may be suitable for children with ASD. Future work will extend the evaluation studies to include children with ASD and more practitioners.
\end{abstract}

\section{KEYWORDS}

Autism, Coping with Changes, Assistive Technology, Interface Design

\section{INTRODUCTION}

Autism Spectrum Disorder (ASD) is a lifelong neurodevelopmental disorder characterized by deficits in social interaction and communication and the appearance of repetitive patterns of behaviour and interests, including resistance to change (APA, 2013). The use of technology-based interventions for ameliorating ASD core deficits has been growing in popularity, and a variety of technologies such as mobile computing, virtual reality or robotics have been introduced to improve social communication skills and support practitioners' work (Grynszpan et. al, 2014, Morin et al. 2018).

Visual Activity Schedules are often used to help children with ASD cope with the challenges posed by various activities or behaviours during an individual task or throughout the day (Dettmer et al. 2000). For children with autism, they promote independence and predictability, and help with transitioning between activities (Banda et al. 2009). Changes may also be introduced into the routine schedule, which is encouraged to alter constantly, so that children can develop adapting skills (Mesibov et al. 2005). Social Stories ${ }^{\text {TM }}$ - first introduced by Gray (2019) - represent short stories describing certain situations, events or activities which are following a set of criteria. They aim to teach children with learning disabilities of certain situations or events, such as getting dressed, and also prepare them for novel events, such as moving house. A series of applications have been developed to support practitioners in creating and children in working with Visual Activity Schedules (Cramer et al., 2011) and Social Stories ${ }^{\text {TM }}$ (Constantin, 2015).

However, limited technologies are available that can help children with autism cope with changes, and these do not typically incorporate the methods used or recommended by practitioners. Therefore, this project explores how technology can be best designed to help children with autism cope with changes. We designed and developed an app called ChangeIt, which may enhance the work of practitioners in helping children with ASD cope with changes. This paper reports on preliminary work, in which we decided not to involve children with ASD before getting some evidence that the app may be useful and suitable for them. Instead, we involved typically developing (TD) children, as well as practitioners and researchers in ASD and Human-Computer Interaction $(\mathrm{HCI})$ as proxies in the initial design and evaluation of the app. The results of the evaluation were 
very promising, suggesting that the app has potential in helping children with autism cope with changes. However, more studies for the app design and evaluation, involving children with ASD, are necessary until these results can be considered generalisable.

\section{METHODOLOGY}

We used a User-Centered Design (UCD) framework, in which users are involved in every stage of the design process (Norman et al., 1986). However, despite children with autism being the end users of our proposed app, involving them in empirical studies can cause distress, and is moreover very time and resource consuming. Instead, we involved TD children, as suggested by other reports (Frauenberger et al., 2012), because they could bring their age-related expertise in the design of technology with good usability for children. Moreover, we involved HCI experts with general experience in usability, and ASD practitioners and experts, who could advise what is appropriate to help children with ASD cope with changes.

The project followed a methodology which is represented in Figure 1 and is detailed in the next sections.

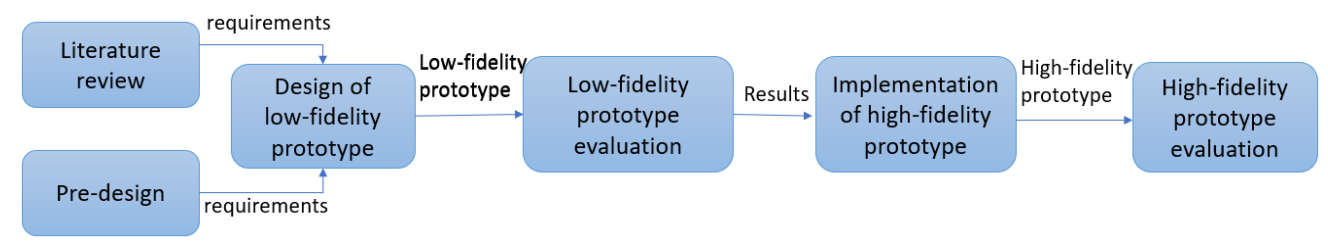

Figure 1. Methodology

\subsection{Pre-Design}

During the pre-design stage, we conducted one-to-one semi-structured interviews with one ASD practitioner and 2 researchers on HCI/ASD with the aim to gather their practical experience with helping children with ASD deal with changes. This stage also involved a workshop using pens and paper with 2 TD children with the aim of identifying the reaction of children to undesirable changes and their strategies to cope with them.

Based on the literature review and interviews, three main methods for helping children with autism cope with changes were identified: Visual Activity Schedules (Dettmer et al., 2000), Social Stories ${ }^{\mathrm{TM}}$ (Gray, 2019) and a list of Coping Strategies. Coping Strategies are used to overcome the difficulties after changes occur and were revealed at the pre-design stage. We decided that our app should support all of these methods.

The literature review and pre-design studies resulted in a list of requirements for the user interface design of the app. Nielsen's 10 Usability Heuristics (Nielsen, 1994) and autism-specific guidelines for user interface design (Bartoli et al, 2014; Pavlov, 2014) helped add requirements to ensure that the app has good usability- both in general and for autistic users. For example, one of the autism-specific guidelines recommends the use of visual cues to improve reading comprehension, and a simple design with contrast between background and font, few and clear elements on screen, and simple graphics (Pavlov, 2014).

Overall, the most important requirements were that the app should:

1. Have two distinct interfaces: one of practitioners and one of children.

2. Allow practitioners to create/edit/delete Social Stories ${ }^{\mathrm{TM}}$, Schedules and Coping Strategies.

3. Allow children to view the Social Stories ${ }^{\mathrm{TM}}$, Schedules and Coping Strategies which had been created by practitioners.

4. Provide simple templates for filling information by practitioners.

5. Provide examples of Social Stories ${ }^{\mathrm{TM}}$, Schedules and Coping Strategies to the practitioner.

6. Include a fictional character, a pet. Using stories with anthropomorphic animals and fantasy can enhance learning, as also suggested by Weisberg et al. (2015).

\subsection{Low-Fidelity Prototype Design and Evaluation}

Using the list of requirements, we designed a low-fidelity prototype using the Figma design tool (Figma, 2019). TD children and experts on HCI/ASD were invited to individually evaluate the low-fidelity prototype. We used 
task-based Evaluation and Cooperative Evaluation (Dix et al., 2004), respectively, followed by a post-task semi-structured interview (for TD children) or online questionnaire (for HCI/ASD experts). The aim was to gather feedback on the app's usability and its suitability for the target population and for helping children with ASD cope with changes. The qualitative data gathered from the studies was analysed using Thematic Analysis (Braun \& Clarke, 2006), following a top-down approach. In terms of usability, results helped identify some issues such as misleading elements of the design and inconsistencies. For example, there was some inconsistency between buttons and the appropriate message after adding/deleting actions. Also, some suggestions for more functionalities were collected (e.g. a reward system to encourage children on ASD to use the app). In terms of suitability, all the experts agreed that the prototype is suitable for autistic children and may help them cope with changes. The app was perceived as easy to use both by children with ASD and practitioners. The evaluation results helped refine the requirements and prototype.

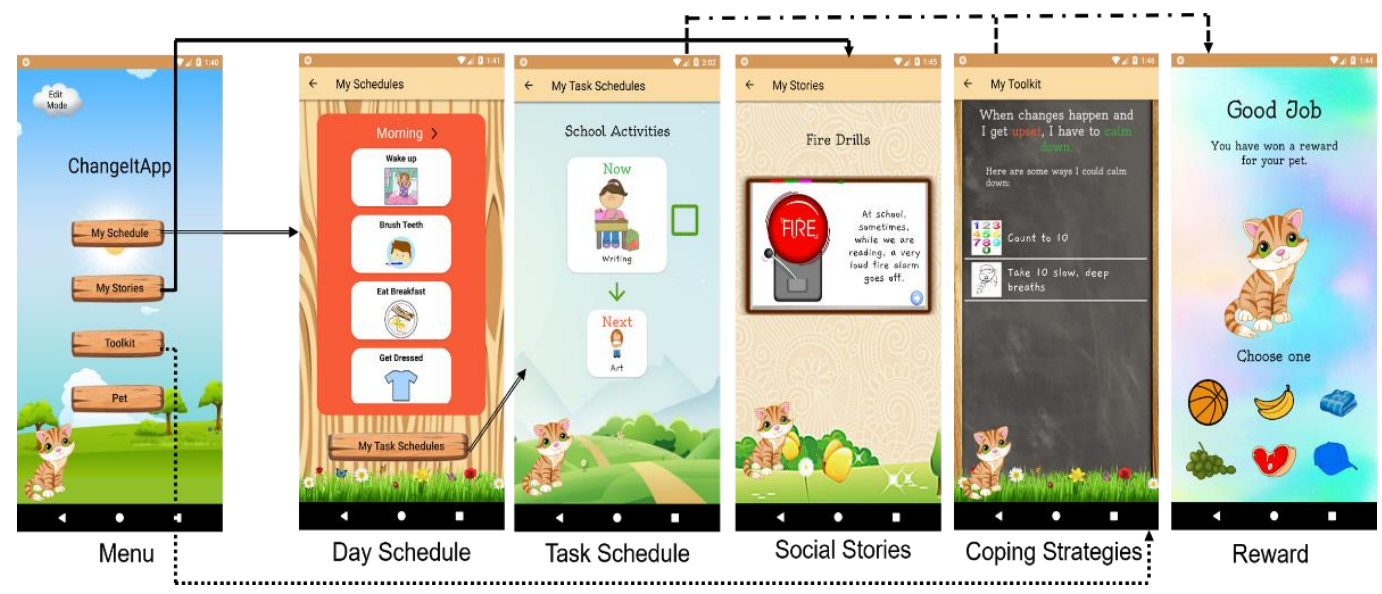

Figure 2. The child interface to the ChangeIt app

\subsection{High-fidelity Prototype Implementation}

A high-fidelity prototype was developed using Android Studio (2019). The child interface opens with the 'Menu' screen which contains three buttons which, when clicked, take the child to either the 'Day Schedule' screen (with the schedule of the day), 'My Stories' (where stories about changes can be read) or the 'Coping Strategy' screen (see Figure 2). In each section, after completing a series of tasks, the child is presented with the 'Rewards Screen' in which they can choose a reward for their pet.

Clicking on the 'Edit mode' button from the Menu Screen leads to the practitioner interface (Figure 3). To create a new task schedule, the practitioner has to add tasks, and to create a new Social Story ${ }^{\mathrm{TM}}$, the practitioner has to add pages. Icons are provided in each screen to add (plus icon), save (floppy disk icon), delete (trash icon) elements. An element can be edited by clicking on it.

\subsection{Evaluation}

In the evaluation stage for the high-fidelity prototype, we first conducted a study with students and staff from the University of Edinburgh, with the aim of assessing the app's general usability. We first asked university students and staff to freely interact with the app and fill in a small online questionnaire consisting of a combination of Likert scale questions and optional open-ended questions. The data was analysed using Descriptive Statistics and Thematic Analysis (Braun \& Clarke, 2006). The majority of the participants agreed that the app is easy to use and suitable for the target population. The app was rated overall as 'Very Good' $(4 / 5)$ or 'Excellent' $(5 / 5)$. A participant mentioned that ChangeIt is a brilliant idea and she suggested that it should be tested further and released to the market. 


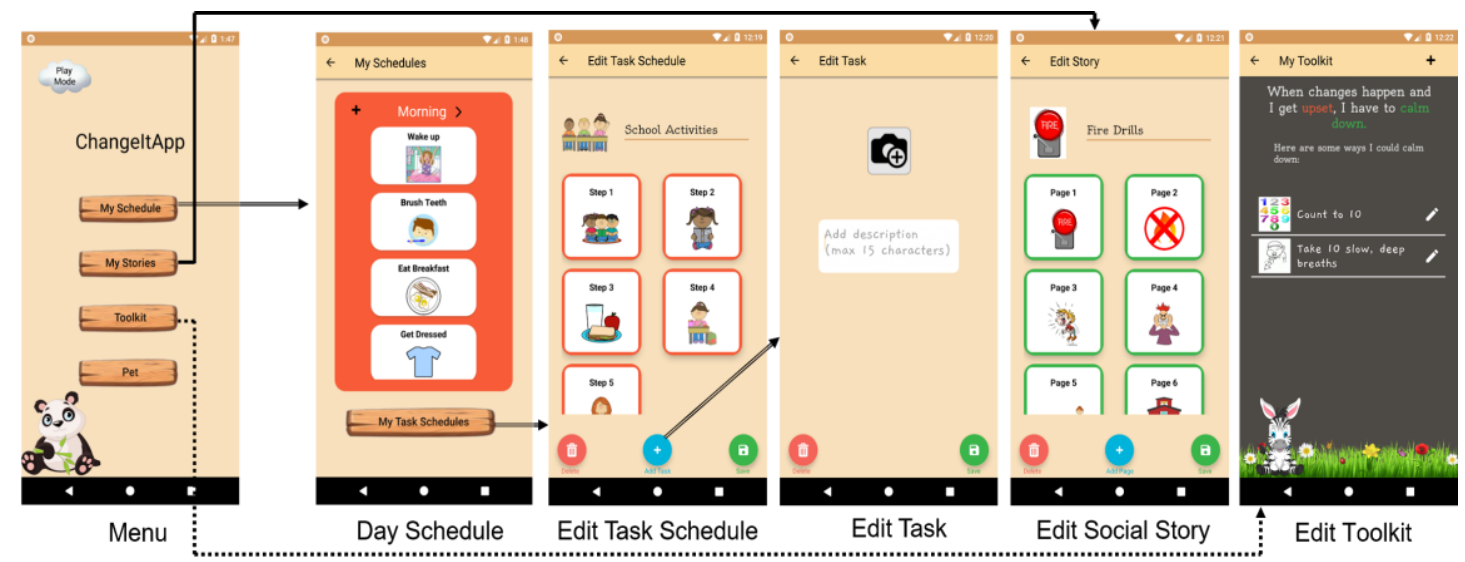

Figure 3. The practitioner interface to the ChangeIt app

We then conducted a study with 5 TD children ( $3 / 5$ having also participated to the low-fidelity prototype evaluation), with the aim of assessing the ChangeIt app's suitability, whether the app's activities are fun and enjoyable, and the children's perceptions of its usability. The study consisted of a combination of task-based Evaluation and free exploration followed by a post-task semi-structured interview, and involved children individually or in pairs. The qualitative data was analysed using Thematic Analysis (Braun \& Clarke, 2006). All of the children found the ChangeIt app very easy to use and its activities fun. The majority (4/5) of the children rated the app as 'Excellent' and for the majority (3/4) the favourite functionality was the reward system. One of the children commented liking that the pet was present in every interface.

Finally, we conducted a study with 6 experts on HCI/ASD, with the aim of assessing the app's suitability and the experts' perceptions of its usability. The study consisted of a combination of Cooperative Evaluation (Dix et al. 2004) followed by a small questionnaire. The data was analysed using Thematic Analysis (Braun \& Clarke, 2006) and Descriptive Statistics. All the experts found the ChangeIt app easy to use and suitable for the target population. A practitioner remarked: "I think it is really easy to use....but I think there are some little things that can be added and enhance the usability of the app, overall. But I don't think it is difficult at all, it's not.". They found the methods used in the app effective in helping children with autism cope with changes. However, 3 out of the 6 experts expressed some doubts with regards to the appropriateness of Coping Strategies. One of them remarked: "When they are upset, the children will not really go and look at something to calm them down". On the other hand, another expert mentioned: "I think you have to train them to go and grab this toolkit. I think this is a great way for them, to remind them what they could do if they get upset". This was a controversial issue but it was agreed that the specific section needs more functionality.

The most important suggestions that were identified after analysing the data of the studies were:

- Adding more interaction with the pet and a high-level reward system to increase motivation ( $5 / 5$ children, $3 / 6$ experts).

- Adding interaction when clicking on the Coping strategies (5/6 experts). One expert explained: "It ['Coping strategies' page] doesn't say much. So maybe if you could click and say a bit more...Maybe you need to include some audio, maybe an option is to listen to a song he likes or pictures or videos of their favourite cartoons." Also one of the children stated: "Honestly, I think of what I want and that is what calms me down...I want a cat so I might think about a cat or me getting a cat... One of the things could be a picture of a cat down here (as a coping strategy)".

- Making the app more customizable by allowing practitioners to upload images of pets ( $2 / 5$ children, $2 / 6$ experts), videos or music (3/6 experts), choose interactions for the pet as rewards ( 1 child, 1 expert), choose the colour scheme (1/6 experts), as well as provide text-to-speech functionality (1/6 experts). One of the experts remarked: "I think if the child has a pet, they can put their own picture or something like that. I think given more customization it's always a good thing."

- Extending the day schedule to a calendar one, to prepare children for changes in advance (1/6 experts). 


\section{CONCLUSION}

This paper presented a preliminary investigation of how technology can be best designed to help children with ASD cope with changes, which resulted in the development of an Android app called ChangeIt. The app was designed and evaluated by involving HCI/ASD experts, practitioners, TD children and general users, following the UCD framework. The results from its evaluation suggested that ChangeIt has potential for helping children with autism cope with changes, it is easy to use, fun and engaging. Furthermore, they led to a series of suggestions that will be considered for improving the app for children with ASD and practitioners.

Nevertheless, any conclusions should be taken with caution. Although the participation of TD children in the development of technology for children with disabilities is useful and important (Grynszpan et. al, 2014), involving children with ASD and a larger sample size would give clearer outcomes. For our next step, we plan to recruit children with ASD, as well as more ASD practitioners, from schools and organisations for children with ASD from across Edinburgh, United Kingdom.

\section{REFERENCES}

American Psychiatric Association (APA). 2013. Diagnostic and statistical manual of mental disorders (DSM-5®). American Psychiatric Pub.

Android Studio, 2019 - last update, Android Studio. [System Software]. Available: https://developer.android.com/studio/, [2019, 1st May]

Banda, D.R., Grimmett, E. and Hart, S.L. 2009. Activity schedules: Helping students with autism spectrum disorders in general education classrooms manage transition issues. Teaching Exceptional Children, 41(4):16-21.

Bartoli, L., Garzotto, F., Gelsomini, M., Oliveto, L. and Valoriani, M. 2014, June. Designing and evaluating touchless playful interaction for ASD children. In Proceedings of the Interaction design and children 2014: 17-26, ACM.

Braun, V. and Clarke, V. 2006. Using thematic analysis in psychology. Qualitative research in psychology, 3(2): 77-101.

Carol Gray, Social Stories ${ }^{\mathrm{TM}}, 2019$ - last update, Carol Gray, Social Stories ${ }^{\mathrm{TM}}$ [Online]. Available: https://carolgraysocialstories.com/social-stories/what-is-it/, [2019, 1st May]

Constantin, A. 2015. Supporting practitioners in social story interventions: the ISISS Authoring Tool.

Cramer, M., Hirano, S.H., Tentori, M., Yeganyan, M.T. and Hayes, G.R. 2011, May. Classroom-based assistive technology: collective use of interactive visual schedules by students with autism. In CHI, 11:1-10.

Dettmer, S., Simpson, R.L., Myles, B.S. and Ganz, J.B. 2000. The use of visual supports to facilitate transitions of students with autism. Focus on Autism and Other Developmental Disabilities, 15(3):163-169.

Dix, A., Finlay, J., Abowd, G. and Beale, R. 2004. Evaluation techniques. Human-computer interaction.

Figma. 2019 - last update, Figma: the collaborative interface design tool. [Online]. Available: https://www.figma.com/, [2019, 1st May]

Frauenberger, C., Good, J. \& Alcorn, A. 2012, June. Challenges, opportunities and future perspectives in including children with disabilities in the design of interactive technology. In Proceedings of the 11th International Conference on Interaction Design and Children, 367-370, ACM.

Grynszpan, O., Weiss, P.L., Perez-Diaz, F. \& Gal, E. 2014. Innovative technology-based interventions for autism spectrum disorders: a meta-analysis. Autism, 18(4):346-361.

Mesibov, G.B., Shea, V. \& Schopler, E. 2005. The TEACCH approach to autism spectrum disorders. Springer Science $\&$ Business Media.

Morin, K. L., Ganz, J. B., Gregori, E. V., Foster, M. J., Gerow, S. L., Genç-Tosun, D., \& Hong, E. R. 2018. A systematic quality review of high-tech AAC interventions as an evidence-based practice. $A A C, 34(2): 104-117$.

Nielsen, J. 1994, June. Heuristic evaluation. In Usability inspection methods, 25-62, John Wiley \& Sons, Inc.

Norman, D.A. \& Draper, S.W. 1986. User centered system design: New perspectives on HCI. CRC Press.

Pavlov, N. 2014. User interface for people with ASD. Journal of Software Engineering and Applications, 7(02):128.

Weisberg, D.S., Ilgaz, H., Hirsh-Pasek, K., Golinkoff, R., Nicolopoulou, A. \& Dickinson, D.K. 2015. Shovels and swords: How realistic and fantastical themes affect children's word learning. Cognitive Development, 35:1-14. 